\title{
Métodos con metodología, estudios con objetivo
} Methods with Methodology, Studies with Objective

\author{
Isidro Jiménez-Gómez, Universidad Complutense de Madrid \\ Doctor en Comunicación Audiovisual y Publicidad. Profesor Asociado en la \\ Universidad Complutense de Madrid y profesor colaborador de la Universitat Oberta de \\ Catalunya (UOC). Miembro del Grupo de Investigación Complutense GECA (Género, \\ Estética y Cultura Audiovisual). ORCID: 0000-0001-7372-7276
}

Francisco José García-Ramos, Universidad Complutense de Madrid

Profesor en el Departamento de Ciencias de la Comunicación Aplicada de la Facultad de Ciencias de la Información de la Universidad Complutense de Madrid. Miembro del Grupo de Investigación Complutense GECA (Género, Estética y Cultura Audiovisual) y de UNESCO-UniTWIN Network on Gender, Media, and ICTs.

ORCID: 0000-0002-1805-650X

"El estudio de cualquier experiencia humana ha de ser multifacético [...] no es pertinente religar aquello que nunca ha estado desligado"

Chavarría-González (2011, p. 27)

Si bien es cierto que la madurez epistemológica de las ciencias ha sido posible por la especialización, el tiempo nos ha ido mostrando que las herramientas metodológicas cuentan justamente con un dinamismo que les permite ser reconstruídas y funcionar en los distintos territorios del conocimiento. Desde esta perspectiva, dejan de tener sentido las viejas disputas entre el paradigma cuantitativo y el cualitativo, de la misma forma que no es mejor un martillo que un destornillador. Sencillamente, tienen funciones distintas.

Aunque los "métodos rígidos" (Della Porta y Keatings, 2013, p. 16) del paradigma cuantitativo han terminando ocupando buena parte del taller de las ciencias experimentales, la metodología cualitativa sigue siendo la clave de las fases "más creativas" -por ejemplo, la fase de "exploración" de Baxter y Babbie, (2004)- y se hace imprescindible en el cuestionamiento del objeto de estudio. En definitiva, la metodología de la investigación no debe subordinar un paradigma al otro, sino articularlos y combinarlos "en función de cual sea el objetivo concreto a cubrir" (Cea D'Ancona, 1998, p. 25).

Este camino hacia la soñada combinación metodológica se enfrenta, sin embargo, a la especificidad operatoria de cada ciencia y, en algunos casos, al papel de sus sujetos operatorios, como bien indica el filósofo Gustavo Bueno (1995). Los métodos son parte material y constitutiva de cada ciencia y no es de extrañar que su propio desarrollo sea una cuestión interna y clave para la subsistencia de la disciplina. De ahí la dificultad de 
participar en la producción científica como sujeto operatorio sin echar mano de la caja de herramientas colectiva y su sesgo cuantitativo en el caso de las ciencias experimentales o su sesgo cualitativo en el caso de las sociales. Dicho sesgo se traduce en una mejor dotación de la caja con herramientas de un tipo u otro, hasta el punto de que la propia caja hace al profesional, de la misma forma que el taller del maestro artesano construía profesionalmente a sus discípulos.

La investigación española es un buen espejo de estas limitaciones metodológicas. Aunque en los últimos años se percibe un creciente interés por aplicar el diseño metodológico cuantitativo a los estudios sobre comunicación (Martínez Nicolás y Saperas Lapiedra, 2016), Nó Sánchez y Muñiz Velázquez (2013) documentan el reducido porcentaje de investigaciones sobre comunicación con metodología experimental en las revistas españolas hasta el punto de hablar de dos estadios epistemológicos diferentes: "la capacidad predictiva a la que toda ciencia debe aspirar, está aún lejos de alcanzarse en España", señalan (Nó Sánchez y Muñiz Velázquez, 2013, p. 129).

También Martínez Nicolás y Saperas Lapiedra señalaban en 2011 que buena parte de la investigación en comunicación en España "se realiza sin atender a procedimientos estandarizados y confiables para obtener los datos e informaciones en que se apoya" (2011, p. 116) y proponían "activar las alarmas sobre la calidad de la investigación que estamos haciendo" (2011, p. 123). Sin embargo, reconocen los autores posteriormente, en prácticamente una década se ha dado un cambio apreciable de la calidad metodológica de la investigación empírica: "entre el 30\% y el 45\% de los artículos publicados en la década de 1998-2007 presentaba carencias metodológicas básicas, mientras que para el conjunto de este periodo más reciente [2008-2014] ese porcentaje se sitúa en el 16\%" (Martínez Nicolás y Saperas Lapiedra, 2016, p. 23).

Con todo, avisan estos autores, es sintomático el "escaso esfuerzo investigador dedicado a la reflexión teórico-conceptual y a la discusión y propuesta de procedimientos metodológicos para la investigación empírica" (Martínez Nicolás y Saperas Lapiedra, 2016, p. 24). Sin ello, argumentan, se resienten las funciones de clarificación conceptual y de propuesta de procedimientos técnicos, necesarias para hacer avanzar la investigación en una dirección epistemológicamente productiva y válida.

Como resultado principal de este déficit en la entidad de la metodologías de la investigación, la clásica distinción entre objetivos y herramientas se diluye: si la metodología no se contruye a partir del objeto de la indagación empírica, las herramientas pierden su posición y sentido dentro de la caja de cada ciencia. De hecho, la tendencia a presentar unas herramientas de análisis o de muestreo específicas como si fueran en sí mismas la metodología de la investigación es un error que comparten las metodologías de corte cualitativo y cuantitativo.

De la misma forma que se confunde en ocasiones el análisis de discurso, la entrevista en profundidad o el grupo focal con el diseño metodológico de tipo cualitativo, el muestreo con aspiración de representatividad se instaura como símbolo inequívoco del correcto diseño de corte cuantitativo, hasta el punto de pretender sustituir dicho diseño. En realidad, el muestreo solo es una parte del proceso metodológico y de nada sirve si no funciona bajo un diseño previo del mismo y, ni siquiera cuando está bien realizado en el 
sentido técnico y se logra una muestra representativa, podemos asegurar que cumpla el objetivo para el que fue utilizado.

Por tanto, y a pesar de los distintos enfoques existentes sobre el papel que en la investigación debe tener el diseño metodológico, parece evidente que las herramientas o técnicas específicas son instrumentos que solo funcionan cuando el propio diseño metodológico así lo pide. Poner en valor el papel de una fase de diseño metodológico en la que se "deberán tomar varias decisiones relacionadas con los métodos que se utilizarán para estudiar el problema de investigación" (Igartua Perosanz, 2006, p. 86) es, en realidad, reconectar con el problema epistemológico a resolver: al fin y al cabo, los objetivos de la investigación son la guía que marca el camino a seguir en todos los pasos del proceso investigador, incluidas lógicamente, las fases de desarrollo técnico.

El primer volumen de la Revista Comunicación \& Métodos reúne artículos sobre temáticas tan diversas como los personajes del relato cinematográfico, la Responsabilidad Social Empresarial en atletas de élite, la visibilidad en Internet de las instituciones universitarias, la intención de compra en las comunidades de compra social, los estudios históricos de la comunicación social, el desorden comunicativo en el arte contemporáneo y el análisis cualitativo de datos en Twitter.

Todos estos artículos proponen un análisis de herramientas específicas a esas temáticas $\mathrm{y}$, a la vez, aportan una perspectiva de conjunto de la riqueza metodológica con la que contamos en el campo de la comunicación. Nuestro humilde propósito con la edición de este primer número de Comunicación y Métodos es aportar un granito de arena en ese camino, reivindicar la metodología más allá de sus métodos.

\section{Referencias}

Baxter, L. A., \& Babbie, E. (2004). The Basics of Communication Research, Belmont: Wadsworth/Thomson Learning.

Bueno, G. (1995). ¿Qué es la ciencia? La respuesta de la teoría del cierre categorial. Ciencia y Filosofía, Oviedo: Pentalfa.

Cea D'Ancona, M. A. (1998). Metodología cuantitativa. Estrategias y técnicas de investigación social ( $3^{\mathrm{a}}$ ed.), Madrid: Síntesis.

Della Porta, D., \& Keating, M. (eds.) (2013). Enfoques y metodologías de las ciencias sociales. Una perspectiva pluralista, Madrid: Akal.

Igartua Perosanz, J. J. (2006). Métodos cuantitativos de investigación en comunicación, Barcelona: Bosch.

Martínez Nicolás, M., \& Saperas Lapiedra, E. (2011). La investigación sobre Comunicación en España (1998-2007). Análisis de los artículos publicados en revistas científicas, Revista Latina de Comunicación Social, 66, 1-129. 
Martínez Nicolás, M., \& Saperas Lapiedra, E. (2016). Objetos de estudio y orientación metodológica de la reciente investigación sobre comunicación en España (20082014), Revista Latina de Comunicación Social, 71, 1.365 - 1.384.

Nó Sánchez, J., \& Muñiz Velázquez, J. A. (2013). El estado de la metodología experimental en la investigación en comunicación, En Investigar la Comunicación hoy. Revisión de políticas científicas y aportaciones metodológicas: Simposio Internacional sobre Política Científica en Comunicación, Facultad de Ciencias Sociales, Juridicas y de la Comunicación, 123-132.

HOW TO CITE (APA)

Jiménez-Gómez, I., \& García-Ramos, F. J. (2019). Métodos con metodología, estudios con objetivo. Comunicación y Métodos | Communication \& Methods, 1(1), 5-8. https://doi.org/10.35951/v1i1.21 